\title{
From Crossing-Free Graphs on Wheel Sets to Embracing Simplices and Polytopes with Few Vertices
}

\author{
Alexander Pilz ${ }^{* 1}$, Emo Welzl ${ }^{2}$, and Manuel Wettstein ${ }^{3}$ \\ 1 Department of Computer Science, ETH Zürich, Zürich, Switzerland \\ pilza@inf.ethz.ch \\ 2 Department of Computer Science, ETH Zürich, Zürich, Switzerland \\ emo@inf.ethz.ch \\ 3 Department of Computer Science, ETH Zürich, Zürich, Switzerland \\ mw@inf .ethz.ch
}

\begin{abstract}
A set $P=H \cup\{w\}$ of $n+1$ points in the plane is called a wheel set if all points but $w$ are extreme. We show that for the purpose of counting crossing-free geometric graphs on $P$, it suffices to know the so-called frequency vector of $P$. While there are roughly $2^{n}$ distinct order types that correspond to wheel sets, the number of frequency vectors is only about $2^{n / 2}$.

We give simple formulas in terms of the frequency vector for the number of crossing-free spanning cycles, matchings, w-embracing triangles, and many more. Based on these formulas, the corresponding numbers of graphs can be computed efficiently.

Also in higher dimensions, wheel sets turn out to be a suitable model to approach the problem of computing the simplicial depth of a point $w$ in a set $H$, i.e., the number of simplices spanned by $H$ that contain $w$. While the concept of frequency vectors does not generalize easily, we show how to apply similar methods in higher dimensions. The result is an $O\left(n^{d-1}\right)$ time algorithm for computing the simplicial depth of a point $w$ in a set $H$ of $n d$-dimensional points, improving on the previously best bound of $O\left(n^{d} \log n\right)$.

Configurations equivalent to wheel sets have already been used by Perles for counting the faces of high-dimensional polytopes with few vertices via the Gale dual. Based on that we can compute the number of facets of the convex hull of $n=d+k$ points in general position in $\mathbb{R}^{d}$ in time $O\left(n^{\max \{\omega, k-2\}}\right)$ where $\omega \approx 2.373$, even though the asymptotic number of facets may be as large as $n^{k}$.
\end{abstract}

1998 ACM Subject Classification G.2.1 Combinatorics, F.2.2 Nonnumerical Algorithms and Problems

Keywords and phrases geometric graph, wheel set, simplicial depth, Gale transform, polytope

Digital Object Identifier 10.4230/LIPIcs.SoCG.2017.54

\section{Introduction}

Computing the number of crossing-free straight-line drawings of certain graph classes (e.g., triangulations, spanning trees, etc.) on a planar point set is a well-known problem in computational and discrete geometry. While for point sets in convex position many of these numbers have simple closed formulas, it seems difficult to compute them efficiently for a

* A.P. is supported by an Erwin Schrödinger fellowship, Austrian Science Fund (FWF): J-3847-N35. 
given general point set, or to provide tight upper and lower bounds. In this work, we provide means for solving these problems for a special class of point sets which we call wheel sets.

Let $P=H \cup\{w\}$ be a set of $n+1$ points in the plane. Unless stated otherwise, $P$ is assumed to be in general position and the points in $H$ are assumed to be extreme (i.e., part of the boundary of the convex hull of $P$ ). $P$ is in convex position if all points including $w$ are extreme, and $P$ is a wheel set if all points except $w$ are extreme. If $P$ is either of them, then we call it a conowheel set. We denote by $P_{\text {con }}$ a concrete set in convex position (say, the vertex set of a regular $(n+1)$-gon) and by $P_{\text {bar }}$ a barely-in wheel set (i.e., $H$ is the vertex set of a regular $n$-gon and $w$ is sufficiently close to an edge $e$ of the $n$-gon in such a way that $w$ is in the interior of every triangle spanned by $e$ and a third point of $H$ ).

The numbers of triangulations and pseudo-triangulations on wheel sets [24], as well as perfect matchings [26], have been studied before. Our work generalizes these approaches. Wheel sets have also been used to represent vectors in the investigation of high-dimensional polytopes with few vertices; already in the 1960s, Perles counted the number of combinatorially different wheel sets (as reported by Grünbaum [15]). In the terminology of modern discrete geometry, these correspond to the different order types of wheel sets.

Order types. The order type of a point set $P$ is a combinatorial description that assigns an orientation (either clockwise or counterclockwise) to every ordered triple of points. Two point sets are said to have the same order type if there exists a bijection between the two sets that preserves these orientations [13]. We follow the practice of considering two point sets to have the same order type if there exists a bijection that reverses all orientations.

Many combinatorial properties of a point set can be recovered from its order type. In particular, the order type determines whether two segments with endpoints in $P$ cross, and whether a given point in $P$ is extreme. It is not hard to see that all sets in convex position have the same order type. However, the same is not true for wheel sets.

- Theorem 1. The number of distinct order types of conowheel sets of size $n+1 i s^{1}$

$$
\frac{1}{4 n} \sum_{2 \nmid k \mid n} \varphi(k) 2^{n / k}+2^{\lfloor(n-3) / 2\rfloor}=\Theta\left(2^{n} / n\right) \text {. }
$$

The above formula has been obtained first by Perles (as stated, without proof, in [15, Chapter 6.3]) for the number of simplicial polytopes with few vertices, and we explain the connection to wheel sets in Section 4. Perles also counted the number of equivalent so-called distended standard forms of Gale diagrams, which basically correspond to wheel sets with different order types. In Section 2 we describe this correspondence.

Frequency vectors. While the order type of a point set determines the set of crossing-free geometric graphs on it, we show in Section 3 that we can rely on the following, coarser classification when only considering wheel sets.

Let $P=H \cup\{w\}$ be a conowheel set and let $h \in H$ be arbitrary. Let $\mathrm{I}(h)$ denote the number of points strictly to the left of the directed line going from $w$ to $h$, and let $r(h)$ denote the number of points strictly to the right of that line. The frequency vector of $P$ is the vector $F(P)=\left(F_{0}, F_{1}, \ldots, F_{n-1}\right)$ where $F_{i}$ is the number of points $h \in H$ satisfying $|\mathrm{I}(h)-\mathrm{r}(h)|=i$. Consider the following examples for $n=7$.

$$
F\left(P_{\text {con }}\right)=(1,0,2,0,2,0,2) \quad F\left(P_{\text {bar }}\right)=(1,0,2,0,4,0,0)
$$

1 Here, $\varphi(k)$ denotes Euler's totient function, which counts the integers coprime to $k$ that are at most $k$. 
Note that the frequency vector can be computed in $O(n \log n)$ time by radially sorting $H$ around $w$. It is also clear that the order type determines the frequency vector. However, the opposite is not true. In Section 2, we give a complete characterization of frequency vectors, which allows us to conclude the following.

Theorem 2. For any $n \geq 1$, the number of frequency vectors realizable by a conowheel set over $n+1$ points is exactly $2^{\lceil n / 2\rceil-1}$.

Given that the number of frequency vectors is significantly smaller than the number of order types, it is unclear how much the frequency vector reveals about a conowheel set. However, we will show that for the purpose of counting crossing-free structures it is both sufficient and necessary.

Moreover, there is again a connection to simplicial polytopes with few vertices. In Section 4 , we show that the number of frequency vectors is equal to the number of $f$-vectors of polytopes in $d$-space with at most $d+3$ vertices (including the empty polytope). The latter has been calculated by Linusson [17] using a sophisticated counting of so-called $M$-sequences.

Geometric graphs. A geometric graph on $P$ is a graph with vertex set $P$ and edges drawn as straight segments between the corresponding endpoints, and it is crossing-free if no two edges intersect in their respective relative interiors.

There exists a vast literature that is concerned with counting these crossing-free structures on specific point sets or proving extremal upper and lower bounds [3, 27, 28, 29]. One comparatively simple case is if $P$ is in convex position. In that case, counting triangulations is a classical problem that goes back to Euler, and it gives rise to the famous Catalan numbers. For many other families of graphs (such as perfect matchings and spanning trees), simple closed formulas can be obtained as well [7, 11, 22].

Randall et al. [24] were the first to consider geometric graphs on wheel sets. They found the extremal configurations for triangulations and pseudo-triangulations by using an argument that involves continuously moving the extra point $w$. The case of perfect matchings has been studied by Ruiz-Vargas and Welzl [26]. The next theorem is a generalization of a result from their paper.

In the following, let $\mathcal{G}$ be a set of abstract (unlabeled) graphs with $n+1$ vertices, and let $\mathrm{nb}_{\mathcal{G}}(P)$ denote the number of crossing-free geometric graphs on $P$ which are isomorphic to a graph in $\mathcal{G}$. In other words, $\mathrm{nb}_{\mathcal{G}}(P)$ is the number of non-crossing straight-line embeddings of graphs in $\mathcal{G}$ on $P$.

Theorem 3. thmggraph Let $\mathcal{G}$ be arbitrary, and let $P=H \cup\{w\}$ be a conowheel set of size $n+1$. Then, $\mathrm{nb}_{\mathcal{G}}(P)$ depends only on the frequency vector $F(P)=\left(F_{0}, F_{1}, \ldots, F_{n-1}\right)$. More concretely,

$$
\mathrm{nb}_{\mathcal{G}}(P)=\gamma_{n}-\frac{1}{2} \sum_{h \in H} \lambda_{\mathrm{I}(h), \mathrm{r}(h)}=\sum_{k=0}^{n-1} F_{k} \Lambda_{k}
$$

where $\gamma_{n}$ and $\lambda_{l, r}=\lambda_{r, l}$ are integers and $\Lambda_{k}$ are rationals depending on $\mathcal{G}$.

While the latter formula in the above theorem makes the dependency on the frequency vector more obvious, the former will turn out to be more natural. The latter formula follows from the former simply by putting $\Lambda_{k}=\gamma_{n} / n+1 / 2 \cdot \lambda_{(n+k-1) / 2,(n-k-1) / 2}$.

We give just one example here, which at the same time makes the connection to the later parts of the paper. Let $\mathcal{G}=\left\{K_{4}^{\cdots}\right\}$, where $K_{4}^{\cdots}$ is obtained by adding $n-3$ additional isolated 
vertices to the complete graph $K_{4}$. The following formula is obtained alongside Theorem 3 in Section 3.

$$
\mathrm{nb}_{\mathcal{G}}(P)=\left(\begin{array}{l}
n \\
3
\end{array}\right)-\frac{1}{2} \sum_{h \in H}\left(\left(\begin{array}{c}
\mathrm{I}(h) \\
2
\end{array}\right)+\left(\begin{array}{c}
\mathrm{r}(h) \\
2
\end{array}\right)\right) \quad \text { for } \mathcal{G}=\left\{K_{4}^{\cdots}\right\} .
$$

Observe that all non-crossing embeddings of $K_{4}^{\cdots}$ on a given conowheel set $P=H \cup\{w\}$ have the following property. One of the vertices of the underlying $K_{4}$ is mapped to the point $w$, while the other three vertices are mapped to three points which form a triangle that contains the extra point $w$ in its interior. We thus get a rather simple formula for the number of w-embracing triangles (i.e., point triples in $H$ whose convex hull contains $w$ ). Note that the set of $w$-embracing triangles does not change if we replace a point $p \in H$ by a point $p^{\prime}$ on the ray starting at $w$ and passing through $p$; for counting $w$-embracing triangles, the approach for conoweel sets thus generalizes to arbitrary point sets. In other words, the formula in equation (1) also counts the number of $w$-embracing triangles in an arbitrary point set $H$ in general position. We note that the algorithm which counts $w$-embracing triangles in $[25]$ is essentially an implementation of equation (1).

Higher dimensions. The concept of conowheel sets can be generalized to arbitrary dimensions, where we may again consider sets with at most one non-extreme point. However, even for counting $w$-embracing tetrahedra in 3-space, the ideas from the proof of Theorem 3 do not generalize. Nevertheless, in Section 4 we give a generalization of equation (1). From that we obtain improved time bounds for computing the number of $w$-embracing simplices or, in other words, the simplicial depth of a point $w$ (as defined in [18]).

- Theorem 4. thmsimpldepth Let $d \geq 3$ be fixed and let $H$ be a set of $n$ points in $\mathbb{R}^{d}$. Then, the simplicial depth of a point $w$ in $H$ can be computed in $O\left(n^{d-1}\right)$ time.

Again, this result is stated for arbitrary sets $H$ and not for wheel sets only, as for the simplicial depth only the position relative to $w$ is relevant. We further note that the algorithm generalizes to counting all $k$-element subsets of $H$ whose convex hull contains $w$.

The simplicial depth of a point has attracted considerable attention as a measure of data depth. Several authors describe the calculation of the simplicial depth of a point in the plane [12, 16, 25]. $O\left(n^{2}\right)$ and $O\left(n^{4}\right)$ time algorithms for 3- and 4-space, respectively, are provided by Cheng and Ouyang [6], who also point out flaws in previous algorithms in 3-space. Our result improves over the previously best known general $O\left(n^{d} \log n\right)$ time algorithm for points in constant dimension $d$ [2]. For arbitrary dimensions, the problem is known to be \#P-complete and $W[1]$-hard [2].

The work by Perles aimed at counting the number of facets of high-dimensional simplicial polytopes with few vertices. Via the Gale dual, this number corresponds to the number of simplices embracing the origin in a dual point set. In Section 4, we show how to compute the number of facets of the convex hull of $d+k$ points in general position in $\mathbb{R}^{d}$ in time $O\left(n^{\max \{\omega, k-2\}}\right)$ (with $O\left(n^{\omega}\right)$ being an upper bound for matrix multiplication).

\section{Order Types and Frequency Vectors}

The purpose of this section is to give an explanation for Table 1. The latter contains the numbers of distinct order types and frequency vectors corresponding to conowheel sets of size $n+1$. For completeness, we have also included the corresponding numbers if equivalence over order types is defined to not include reflections. 
Table 1 Number of order types and frequency vectors of conowheel sets over $n+1$ points.

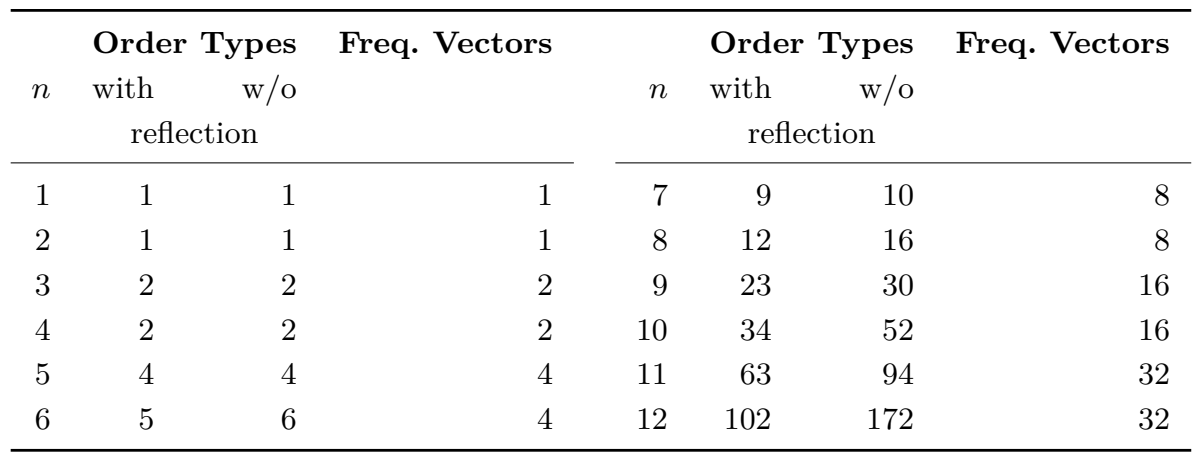
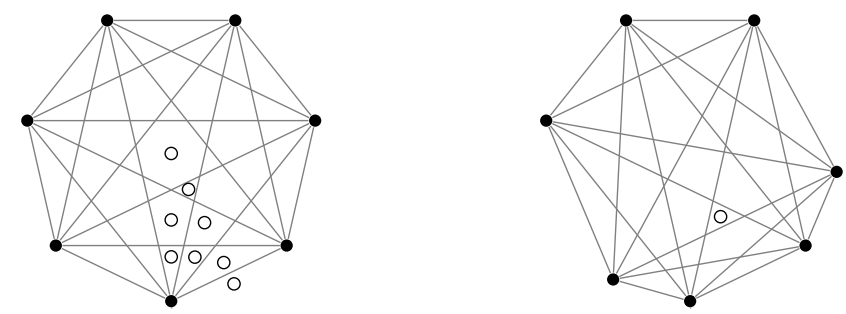

Figure 1 All order types of conowheel sets for $n=7$. The extra point $w$ is drawn in white.

Order types. Given a set $H$ of $n=7$ points forming the vertex set of a regular heptagon, there are 8 conowheel sets $P=H \cup\{w\}$ with distinct order types that can be obtained by adding an extra point $w$, see the left hand side of Figure 1. Notice the discrepancy with the number 9 displayed in Table 1. The ninth and last order type can be obtained by first deforming $H$ as illustrated on the right hand side of Figure 1. This necessary deformation of $H$ seems to complicate matters significantly, but only at first sight.

- Theorem 1 (restated). The number of distinct order types of conowheel sets of size $n+1$ $i s^{2}$

$$
\frac{1}{4 n} \sum_{2 \nmid k \mid n} \varphi(k) 2^{n / k}+2^{\lfloor(n-3) / 2\rfloor}=\Theta\left(2^{n} / n\right) .
$$

The asymptotic estimate is explained by taking the dominant summand with $k=1$. The exact formula was first obtained by Perles (cf. [15, Chapter 6.3]) as the number of combinatorially different simplicial polytopes in dimension $n-3$ with at most $n$ vertices.

The formula was also obtained in the context of counting the number of 2-colored selfdual necklaces with $2 n$ beads with mirrored necklaces identified [5, 23]. These are binary (say, black and white) circular sequences of length $2 n$ such that elements at distance $n$ (i.e., opposing beads) are distinct (i.e., if one is black the other must be white, and vice versa). The correspondence between simplicial polytopes and necklaces has been shown by Montellano-Ballesteros and Strausz [21] using Radon complexes, unaware of Perles' result.

A proof of Theorem 1 can be given by using a simple bijection to necklaces. We note that a similar (and slightly simpler) formula is known if mirrored necklaces are not identified. Naturally, that formula also counts order types of conowheel sets without reflection.

2 Here, $\varphi(k)$ denotes Euler's totient function, which counts the integers coprime to $k$ that are at most $k$. 

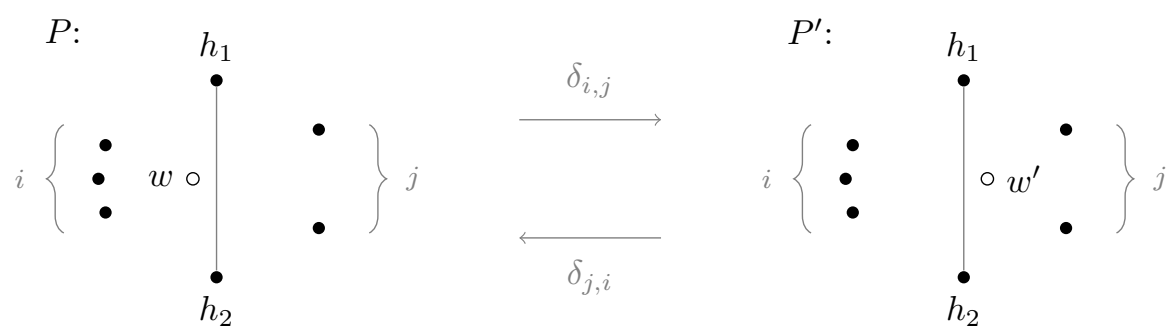

Figure 2 Moving the extra point over the segment $h_{1} h_{2}$ for the case $i=3$ and $j=2$.

Frequency vectors. The following lemma gives a characterization of frequency vectors. The proof is by letting a line rotate about the extra point $w$, and by observing how it dissects the point set $H$ during the process. Full details can be found in [26].

- Lemma 5. $F=\left(F_{0}, F_{1}, \ldots, F_{n-1}\right) \in \mathbb{N}^{n}$ is the frequency vector of a conowheel set $P=H \cup\{w\}$ of size $n+1$, i.e., $F=F(P)$, if and only if

(i) $\sum_{k=0}^{n-1} F_{k}=n$,

(ii) $F_{k}=0$ for all $k \equiv_{2} n$,

(iii) $F_{k}$ is even for all $k \geq 1$, and

(iv) if $F_{k} \neq 0$ and $k \geq 2$, then $F_{k-2} \neq 0$.

With this characterization, it is not hard to determine the number of frequency vectors.

- Theorem 2 (restated). For any $n \geq 1$, the number of frequency vectors realizable by a conowheel set over $n+1$ points is exactly $2^{\lceil n / 2\rceil-1}$.

Proof. For $n=1$ and $n=2$ the formula evaluates to 1 , which is consistent with the fact that there is only one respective order type for either two or three points. For larger $n$, we give a proof by induction for odd $n$, and note that even $n$ can be handled analogously.

So let $n=m+2 \geq 3$ be odd. Using Lemma 5 , we can characterize the set of frequency vectors that are realizable by $n+1$ points by saying that it contains all vectors $F=$ $\left(F_{0}, F_{1}, \ldots, F_{n-1}\right)$ which have one of the following two mutually exclusive forms.

- $F_{0}=1, F_{1}=0$, and $\left(F_{2}-1, F_{3}, F_{4}, \ldots, F_{n-1}\right)$ is any frequency vector realizable by $m+1$ points.

- $F_{0} \geq 3$ is odd, $F_{n-2}=F_{n-1}=0$, and $\left(F_{0}-2, F_{1}, F_{2}, \ldots, F_{n-3}\right)$ is any frequency vector realizable by $m+1$ points.

If $2^{\lceil m / 2\rceil-1}$ is the number of frequency vectors realizable by $m+1$ points, the corresponding number for $n+1$ points is thus $2 \cdot 2^{\lceil m / 2\rceil-1}=2^{\lceil n / 2\rceil-1}$.

\section{Geometric Graphs}

Recall that $\mathrm{nb}_{\mathcal{G}}(P)$ is the number of crossing-free geometric graphs on a point set $P$ that are isomorphic to a graph in the family $\mathcal{G}$. Consider now two conowheel sets $P=H \cup\{w\}$ and $P^{\prime}=H \cup\left\{w^{\prime}\right\}$ which can be transformed into each other by moving the extra point over the segment between $h_{1}, h_{2} \in H$ (and without encountering any other collinearities). The situation is illustrated in Figure 2. Assume that on the $w$-side of the segment $h_{1} h_{2}$ there are $i$ points of $H$, and on the $w^{\prime}$-side there are $j$ points of $H$ (thus, $i+j=n-2$ ). Let $\delta_{i, j}$ be the increment of $\mathrm{nb}_{\mathcal{G}}$ when going from $P$ to $P^{\prime}$, i.e., $\delta_{i, j}=\mathrm{nb}_{\mathcal{G}}\left(P^{\prime}\right)-\mathrm{nb}_{\mathcal{G}}(P)$. 
- Lemma 6. For every $\mathcal{G}, \delta_{i, j}$ is well-defined, i.e., its value depends only on $i, j$ and $\mathcal{G}$, and not on the exact placement of $H$ or the location where the extra point traverses the segment between $h_{1}$ and $h_{2}$.

Proof. All geometric graphs that do not contain the edge $\left\{h_{1}, h_{2}\right\}$ are not affected by the mutation, i.e., they are crossing-free on $P$ if and only if they are crossing-free on $P^{\prime}$. Therefore, $\delta_{i, j}$ is equal to the number of crossing-free geometric graphs on $P^{\prime}$ containing $\left\{h_{1}, h_{2}\right\}$ minus the number of crossing-free geometric graphs on $P$ containing $\left\{h_{1}, h_{2}\right\}$. For the following reasons, these numbers only depend on $i, j$ and $\mathcal{G}$.

In the case of $P$, on the $w$-side we have $i+3$ points (including $h_{1}$ and $h_{2}$ ) in a barely-in configuration, for which there exists a unique order type. On the opposite side we have $j+2$ points (including $h_{1}$ and $h_{2}$ ) in convex position, for which there also exists a unique order type. Because of the presence of the edge $\left\{h_{1}, h_{2}\right\}$ any other edges must be completely contained in one of the two sides, and the claim follows. In the case of $P^{\prime}$, an analogous argument works.

Example, embracing triangles. Consider the case $\mathcal{G}=\left\{K_{4}^{\cdots}\right\}$. Observe that any crossingfree embedding of $K_{4}^{\cdots}$ on $P$ uses $w$ as the inner vertex of the underlying $K_{4}$. Furthermore, if the embedding uses the edge $\left\{h_{1}, h_{2}\right\}$, which implies that $h_{1}$ and $h_{2}$ are outer vertices of $K_{4}$, then any one of the $i$ points on the left hand side can be used as the third outer vertex of $K_{4}$. This gives exactly $i$ crossing-free embeddings of $K_{4}^{\cdots}$ on $P$ which use the edge $\left\{h_{1}, h_{2}\right\}$. Similarly, we get $j$ for the corresponding number of embeddings on $P^{\prime}$. Therefore, $\delta_{i, j}=j-i$ for $\mathcal{G}=\left\{K_{4}^{\cdots}\right\}$.

Theorem 3 (restated). Let $\mathcal{G}$ be arbitrary, and let $P=H \cup\{w\}$ be a conowheel set of size $n+1$. Then, $\operatorname{nb}_{\mathcal{G}}(P)$ depends only on the frequency vector $F(P)=\left(F_{0}, F_{1}, \ldots, F_{n-1}\right)$. More concretely,

$$
\mathrm{nb}_{\mathcal{G}}(P)=\gamma_{n}-\frac{1}{2} \sum_{h \in H} \lambda_{\mathrm{I}(h), \mathrm{r}(h)}=\sum_{k=0}^{n-1} F_{k} \Lambda_{k},
$$

where $\gamma_{n}$ and $\lambda_{l, r}=\lambda_{r, l}$ are integers and $\Lambda_{k}$ are rationals depending on $\mathcal{G}$.

Proof. We proceed by choosing the numbers $\lambda_{l, r}$ such that the validity of the formula is preserved under continuous motion of $P$, and then choose $\gamma_{n}$ such that it holds for some starting configuration. To be more concrete, we allow continuous motion of $P$ where all points are allowed to move if $P$ is in convex position, and only $w$ is allowed to move if $P$ is a wheel set. At discrete moments in time we allow collinearity of three points, the one in the middle being $w$. In this way any two conowheel sets can be transformed into each other.

Let now $P$ and $P^{\prime}$ be as in Figure 2. Note that the values $\mathrm{I}(h)$ and $\mathrm{r}(h)$ do not change for any $h \in H \backslash\left\{h_{1}, h_{2}\right\}$ when going from $P$ to $P^{\prime}$. For $h_{1}$ and $h_{2}$ the corresponding values are

$$
\begin{array}{lll}
P: & \mathrm{I}\left(h_{1}\right)=\mathrm{r}\left(h_{2}\right)=i & \mathrm{r}\left(h_{1}\right)=\mathrm{I}\left(h_{2}\right)=j+1 \\
P^{\prime}: & \mathrm{I}\left(h_{1}\right)=\mathrm{r}\left(h_{2}\right)=i+1 & \mathrm{r}\left(h_{1}\right)=\mathrm{I}\left(h_{2}\right)=j
\end{array}
$$

We therefore preserve the validity of the formula as long as the numbers $\lambda_{l, r}=\lambda_{r, l}$ are chosen in such a way that the following equality holds.

$$
\delta_{i, j}=\frac{1}{2}\left(\lambda_{i, j+1}+\lambda_{j+1, i}\right)-\frac{1}{2}\left(\lambda_{i+1, j}+\lambda_{j, i+1}\right)=\lambda_{i, j+1}-\lambda_{i+1, j} .
$$

The definition $\lambda_{l, r}:=\delta_{n-2,0}+\delta_{n-3,1}+\cdots+\delta_{l, r-1}+c_{n}$ satisfies this constraint. Moreover, the assumed symmetry $\lambda_{l, r}=\lambda_{r, l}$ follows from the skew-symmetry $\delta_{i, j}=-\delta_{j, i}$. Note that 
$l+r=n-1$ always, and that $c_{n}$ is an arbitrary integer independent of $l$ and $r$ (for the proof to go through one could simply fix $c_{n}=0$ ).

Finally, $\gamma_{n}$ is chosen in such a way that the formula holds for some conowheel set. The most natural choice for "anchoring" the formula is a set in convex position.

$$
\gamma_{n}:=\mathrm{nb}_{\mathcal{G}}\left(P_{\text {con }}\right)+\frac{1}{2} \sum_{l, r: l+r=n-1} \lambda_{l, r}
$$

Computing the frequency vector can be done in $O(n \log n)$ time. Given the values $\Lambda_{k}$, computing the number $\mathrm{nb}_{\mathcal{G}}(P)$ of embeddings then requires only $O(n)$ additional arithmetic operations.

Example continued, embracing triangles. We already derived $\delta_{i, j}=j-i$ for $\mathcal{G}=\left\{K_{4}^{\cdots}\right\}$. This now gives rise to

$$
\lambda_{l, r}=\delta_{n-2,0}+\delta_{n-3,1}+\cdots+\delta_{l, r-1}+c_{n}=\sum_{j=0}^{r-1} j-\left(\left(\begin{array}{c}
n-1 \\
2
\end{array}\right)-\sum_{i=0}^{l-1} i\right)+c_{n}=\left(\begin{array}{l}
l \\
2
\end{array}\right)+\left(\begin{array}{l}
r \\
2
\end{array}\right),
$$

if we choose $c_{n}=\left(\begin{array}{c}n-1 \\ 2\end{array}\right)$. It can be checked that $\operatorname{nb}_{\mathcal{G}}\left(P_{\text {con }}\right)=0$. Hence,

$$
\gamma_{n}=\mathrm{nb}_{\mathcal{G}}\left(P_{\text {con }}\right)+\frac{1}{2} \sum_{l, r: l+r=n-1} \lambda_{l, r}=0+\frac{1}{2} \sum_{l=0}^{n-1}\left(\begin{array}{l}
l \\
2
\end{array}\right)+\frac{1}{2} \sum_{r=0}^{n-1}\left(\begin{array}{l}
r \\
2
\end{array}\right)=\left(\begin{array}{l}
n \\
3
\end{array}\right) .
$$

After putting everything together we obtain the exact formula displayed earlier in equation (1).

\subsection{Further Examples}

We call the following two simple applications "insensitive" since the number of crossing-free embeddings is the same on all wheel sets, but may be different for sets in convex position.

Spanning cycles. Consider the case where $\mathcal{G}$ contains a cycle over $n+1$ vertices. Then, we have $\delta_{i, j}=0$ because no crossing-free spanning cycle can use the edge $\left\{h_{1}, h_{2}\right\}$. That is, unless $i=0$ or $j=0$, in which case we have $\delta_{0, n-2}=-\delta_{n-2,0}=n-1$. For anchoring we calculate $\operatorname{nb}_{\mathcal{G}}\left(P_{\text {con }}\right)=1$. It follows that all wheel sets over $n+1$ points admit $n$ crossing-free spanning cycles (which can easily be seen directly).

Spanning paths. If $\mathcal{G}$ contains a path over $n+1$ vertices we also get $\delta_{i, j}=0$ unless $i=0$ or $j=0$, but for a different reason. On $P$ there are $2 \cdot 2^{i} \cdot 2^{j-1}$ crossing-free embeddings that use the edge $\left\{h_{1}, h_{2}\right\}$, since there are 2 choices for deciding which one of $h_{1}$ and $h_{2}$ is connected to the left hand side, $2^{i}$ choices for completing the left hand side to a path and $2^{j-1}$ choices for completing the right hand side to a path. Likewise, on $P^{\prime}$ there are $2 \cdot 2^{i-1} \cdot 2^{j}$ embeddings, which is the same number. Using a similar argument it can be checked that

$$
\delta_{0, n-2}=-\delta_{n-2,0}=2 \cdot\left(2^{n-2}+\sum_{k=1}^{n-2} 2^{k-1} \cdot 2^{n-2-k}\right)-2 \cdot 2^{n-3}=(n-1) 2^{n-2} .
$$

For anchoring we compute $\mathrm{nb}_{\mathcal{G}}\left(P_{\text {con }}\right)=(n+1) 2^{n-2}$, implying $\mathrm{nb}_{\mathcal{G}}(P)=n 2^{n-1}$ for any wheel set $P$.

The following two applications are "sensitive" in the sense that different wheel sets in general have different numbers of crossing-free embeddings. The running example with embracing triangles also is of this kind. 
Matchings. Let $\mathcal{G}=\mathcal{M}$, the set of (not necessarily perfect) matchings over $n+1$ vertices. It is known that $\mathrm{nb}_{\mathcal{M}}\left(P_{\text {con }}\right)=M_{n+1}:=\sum_{k=0}^{\lfloor(n+1) / 2\rfloor}\left(\begin{array}{c}n+1 \\ 2 k\end{array}\right) C_{k}$, the $(n+1)$-th Motzkin number [22]. It is thus easy to compute $\delta_{i, j}=M_{i} M_{j+1}-M_{i+1} M_{j}$ since, as always, we only have to worry about embeddings that use the edge $\left\{h_{1}, h_{2}\right\}$. This gives $\lambda_{l, r}=M_{l} M_{r}$ and $\gamma_{n}=M_{n+1}+\frac{1}{2} \sum_{l, r} M_{l} M_{r}$. After simplifying ${ }^{3}$, we obtain the following formula.

$$
\mathrm{nb}_{\mathcal{G}}(P)=\frac{3 M_{n+1}-M_{n}}{2}-\frac{1}{2} \sum_{h \in H} M_{\mathrm{l}(h)} M_{\mathrm{r}(h)} \quad \text { for } \mathcal{G}=\mathcal{M}
$$

Spanning trees. Let $\mathcal{G}=\mathcal{S} \mathcal{T}$, the set of all trees over $n+1$ vertices. We will make use of the fact that $\operatorname{nb}_{\mathcal{S T}}\left(P_{\text {con }}\right)=T_{n+1}:=\frac{1}{2 n+1}\left(\begin{array}{c}3 n \\ n\end{array}\right)[7,11]$. Furthermore, we will use the hypergeometric identity $\sum_{k=0}^{i} T_{k+1} T_{i-k+1}=\frac{1}{i+1}\left(\begin{array}{c}3 i+1 \\ i\end{array}\right)$. To compute $\delta_{i, j}$, consider the set $P$ as in Figure 2. In order to complete the left hand side into a spanning tree, we have to build two disjoint trees rooted at $h_{1}$ and $h_{2}$, respectively. There are 2 choices for assigning $w$ either to the upper or the lower tree, and there are $i+1$ choices for distributing the $i$ points on the left among the two trees. Indeed, the latter claim holds because the $k$ out of $i$ points assigned to $h_{1}$, say, have to appear consecutively with $h_{1}$ on the convex hull as otherwise we are forced to create a crossing. Once the points have been distributed, we are left with two point sets of size $k+1$ and $i-k+2$ in convex position. For completing the right hand side into a spanning tree, a simpler argument can be used without the additional complication of $w$. Moreover, the set $P^{\prime}$ can be handled analogously.

$$
\begin{aligned}
\delta_{i, j} & =2 \sum_{k=0}^{j} T_{k+1} T_{j-k+2} \cdot \sum_{k=0}^{i} T_{k+1} T_{i-k+1}-2 \sum_{k=0}^{i} T_{k+1} T_{i-k+2} \cdot \sum_{k=0}^{j} T_{k+1} T_{j-k+1} \\
& =2\left(\frac{2}{j+2}\left(\begin{array}{c}
3 j+3 \\
j
\end{array}\right) \cdot \frac{1}{i+1}\left(\begin{array}{c}
3 i+1 \\
i
\end{array}\right)-\frac{2}{i+2}\left(\begin{array}{c}
3 i+3 \\
i
\end{array}\right) \cdot \frac{1}{j+1}\left(\begin{array}{c}
3 j+1 \\
j
\end{array}\right)\right)
\end{aligned}
$$

For this application, we do not know if a simple closed form expression for $\lambda_{l, r}$ exists. Still, note that if one were to compute $\operatorname{nb}_{\mathcal{S T}}(P)$, the numbers $\delta_{i, j}$ can be summed up in linear time and the value of $\gamma_{n}$ can be computed on the fly for any given $n$.

Other applications. Observe that, for example, a geometric triangulation of $P_{\text {con }}$ can be embedded as a plane graph on $P_{\text {bar }}$. However, this embedding is no longer a triangulation (i.e., a tessellation of $\mathrm{CH}\left(P_{\text {bar }}\right)$ into triangles). Hence, there is no natural choice of $\mathcal{G}$ such that $\mathrm{nb}_{\mathcal{G}}(P)$ is the number of triangulations of any conowheel set $P$. It turns out that there are several other families of geometric graphs (pseudotriangulations, crossing-free convex partitions, etc.) whose quantity on a conowheel set $P$ cannot be expressed easily in the form $\mathrm{nb}_{\mathcal{G}}(P)$, but for which the continuous motion argument is still applicable and leads to similarly simple formulas. All that is required is a specialized version of Lemma 6 .

Furthermore, we note that Theorem 3 generalizes to crossing-free embeddings of hypergraphs, where "crossing-free" means that the convex hulls of any two hyperedges intersect in an at most 1-dimensional set.

\footnotetext{
3 A crossing-free matching on $P_{\text {con }}$ either leaves $w$ unmatched $\left(M_{n}\right.$ possibilities $)$ or it matches $w$ with one of the other $n$ points $\left(\sum_{l, r} M_{l} M_{r}\right.$ possibilities). Hence, as required, $M_{n+1}=M_{n}+\sum_{l, r} M_{l} M_{r}$.
} 


\subsection{Wheel Sets and the Rectilinear Crossing Number}

Even though conowheel sets and the associated frequency vectors seem like a very specific set of objects, they do occur naturally in more general settings. Consider for example an arbitrary set $\tilde{P}$ of $n+1$ points in general position and let $\square$ and $\triangle$ be the number of 4-element subsets of $\tilde{P}$ in convex and in non-convex position, respectively. ${ }^{4}$ Let $p \in \tilde{P}$ be any point. We can construct a conowheel set $P=H \cup\{w\}$ containing $w=p$ and, for every $q \in \tilde{P} \backslash\{p\}$, the point $h$ which lies on the intersection of the ray from $p$ to $q$ and a fixed circle centered at $w$ (as done also, e.g., in [16]). That is, $P$ is simply a representation of the local sequence of $p$ in $\tilde{P}$ in terms of conowheel sets; see [14]. Further observe that a triangle spanned by points in $\tilde{P}$ contains $p$ iff its image in $P$ contains $w$. Hence, $\mathrm{nb}_{K_{4}} \ldots(P)$ is the number of such triangles, which is given by equation (1). We thus obtain $\triangle$ by a summation over all points $p$ in $\tilde{P}$. Since $\square+\triangle=\left(\begin{array}{c}n+1 \\ 4\end{array}\right)$, we also obtain $\square$. We note that this can be transformed into equations from $[1,19,30]$ that give $\square$ in terms of the number of $j$-edges (i.e., directed edges spanned by $\tilde{P}$ with exactly $j$ points of $\tilde{P}$ to their left).

To sum up, we can associate a frequency vector with every point of a given point set, and this set of frequency vectors determines the value of $\square$. Unfortunately, this simple approach does not generalize to counting other types of graphs; there are examples of point sets with the same set of frequency vectors but a different number of triangulations.

\section{$4 \quad$ Higher Dimensions: Origin-embracing Simplices}

As already noted in the introduction, the concept of conowheel sets can be generalized to higher dimensions. However, already in $\mathbb{R}^{3}$ we face certain challenges. For example, the number of tetrahedralizations of $n+1$ points in convex position in $\mathbb{R}^{3}$ does not only depend on $n$, in contrast to the 2-dimensional case. Even when considering simpler structures, like the set of $w$-embracing tetrahedra, the ideas from Section 3 do not generalize. (Intuitively, our argument of $w$ passing over a segment does not work in 3-space, as it can pass "behind" a triangle.) Still, we can use similar techniques to obtain improved time bounds for computing the simplicial depth of a point $w$.

Oriented simplices. Given a set $T$ of $d$ affinely independent points in $\mathbb{R}^{d}$, its convex hull $\mathrm{CH}(T)$ is a $(d-1)$-simplex and its affine hull is a hyperplane. We want to be able to refer to the two sides of this hyperplane by identifying a positive and a negative side. For that consider a sequence $p_{1} p_{2} \ldots p_{d}$ of $d$ affinely independent points. The affine hull of $\left\{p_{1}, p_{2}, \ldots, p_{d}\right\}$ can be described as the set of points $q$ with $\sigma\left(q, p_{1} p_{2} \ldots p_{d}\right)=0$, where $\sigma\left(q, p_{1} p_{2} \ldots p_{d}\right):=$ $\operatorname{det}\left(p_{1}-q, p_{2}-q, \ldots, p_{d}-q\right)$. We call the set of points $q$ with $\sigma\left(q, p_{1} p_{2} \ldots p_{d}\right)>0$ the positive side of $p_{1} p_{2} \ldots p_{d}$, and the set of points $q$ with $\sigma\left(q, p_{1} p_{2} \ldots p_{d}\right)<0$ the negative side of $p_{1} p_{2} \ldots p_{d}$. Note that for $d=2$, the positive side of $p_{1} p_{2}$ is the set of points left of the line through $p_{1}$ and $p_{2}$, directed from $p_{1}$ to $p_{2}$. Also note that the positive side of $p_{1} p_{2}$ coincides with the negative side of $p_{2} p_{1}$. For $d \geq 3$, distinct orderings of the given $d$ points may have identical positive sides; this happens iff the orderings can be obtained from each other by an even number of transpositions. An oriented $(d-1)$-simplex is a sequence $p_{1} p_{2} \ldots p_{d}$ of $d$ affinely independent points, with its associated $(d-1)$-simplex $\mathrm{CH}\left(\left\{p_{1}, p_{2}, \ldots, p_{d}\right\}\right)$, and its associated positive and negative side as defined above. Two such oriented $(d-1)$-simplices

4 The number $\square$ is also the number of crossings of the complete geometric graph on $\tilde{P}$, a quantity that has obtained special attention in connection with the so-called rectilinear crossing number of $K_{n}$ (i.e., the smallest number of crossings in a straight line drawing of the complete graph in the plane). 
are defined to be equivalent if their sequences can be obtained from each other by an even number of transpositions (e.g., $p_{1} p_{2} p_{3} \equiv p_{3} p_{1} p_{2} \equiv p_{2} p_{3} p_{1}$ and $p_{3} p_{2} p_{1} \equiv p_{1} p_{3} p_{2} \equiv p_{2} p_{1} p_{3}$ ).

Via oriented simplices, the concept of order types generalizes to arbitrary dimensions; the order type of a set $P=H \cup\{w\}$ of $n+1$ points in $\mathbb{R}^{d}$ determines the set of points on the positive side of the oriented $(d-1)$-simplex $w h_{1} \ldots h_{d-1}$, for each $(d-1)$-tuple in $H$. Let $\mathrm{I}\left(h_{1} \ldots h_{d-1}\right)$ be the number of these points, and let $\mathrm{r}\left(h_{1} \ldots h_{d-1}\right)=n-d+1-\mathrm{I}\left(h_{1} \ldots h_{d-1}\right)$. We can thus define the frequency vector $F(P)=\left(F_{0}, F_{1}, \ldots, F_{n-d+1}\right)$ by letting $F_{i}$ denote the number of tuples $\rho \in H^{d-1}$ s.t. $|\mathrm{I}(\rho)-\mathrm{r}(\rho)|=i$. However, already for $d=3$ it turns out that this frequency vector does not always determine the number of $w$-embracing tetrahedra, i.e., the number of subsets of $d+1$ points of $H$ whose convex hull contains $w$.

\subsection{Embracing sets}

Here, we generalize the notion of embracing triangles to larger sets. Consider a set $P=$ $H \cup\{w\}$ of $n+1$ points in $\mathbb{R}^{d}$. A subset $A \subseteq H$ is a $w$-embracing $k$-set if $w \in \mathrm{CH}(A)$ and $|A|=k$. For simplicity, we will usually consider $w=\mathbf{0}$ and call $A$ an embracing $k$-set.

Let us quickly return to dimension $d=2$ and consider a conowheel set $P=H \cup\{w\}$. As follows, we can show that the number of embracing $k$-sets is determined by the frequency vector of $P$ for any $k$, and not just for $k=3$ as seen earlier in equation (1). Indeed, since $H$ is in general position, for every non-embracing $k$-set $A \subseteq H$ there exists a unique point $h \in A$ such that $\mathrm{CH}(A)$ is in the closed halfplane to the left of the directed segment $w h$. Observe that for any choice of $h \in H$ we can construct $\left(\begin{array}{l}\mathrm{I}(h) \\ k-1\end{array}\right)$ such non-embracing $k$-sets, and thus we get a generalization of equation (1). (This direct approach to counting non-embracing triangles was, e.g., also used in [25].) Interestingly, the reverse is also true.

- Lemma 7. The sequence $\left(\mathrm{embr}_{k}\right)_{k=3}^{n}$, where $\mathrm{embr}_{k}$ is the number of embracing $k$-sets in a conowheel set $P=H \cup\{w\}$ of size $n+1$, determines the frequency vector of $P$.

Proof. Let $E=\left(\mathrm{embr}_{k}\right)_{k=3}^{n}$. Consider the vector $L=\left(L_{j}\right)_{j=1}^{n-1}$ where $L_{j}$ is the number of points $h \in H$ with $\mathrm{I}(h)=j$. Clearly, $L$ determines the frequency vector of $P$. It thus suffices to show that $E$ determines $L$.

The number embr ${ }_{k}$ of embracing $k$-sets is

$$
\mathrm{embr}_{k}=\left(\begin{array}{l}
n \\
k
\end{array}\right)-\sum_{h \in H}\left(\begin{array}{c}
\mathrm{I}(h) \\
k-1
\end{array}\right),
$$

which may be rewritten as

$$
\left(\begin{array}{l}
n \\
k
\end{array}\right)-\mathrm{embr}_{k}=\sum_{j=1}^{n-1} L_{j}\left(\begin{array}{c}
j \\
k-1
\end{array}\right) .
$$

Observe that the above equation also holds for $k=2$. We can thus define a vector $E^{\prime}=\left(e_{i}\right)_{i=1}^{n-1}$ with $e_{i}=\left(\begin{array}{c}n \\ i+1\end{array}\right)-\mathrm{embr}_{i+1}$ and a square matrix $A=\left(a_{i, j}\right)_{i, j=1}^{n-1}$ with $a_{i, j}=\left(\begin{array}{c}j \\ i\end{array}\right)$, such that

$$
E^{\prime}=A L
$$

Since the matrix $A$ is unitriangular (i.e., triangular and without zeroes on the diagonal) it has an inverse, from which we conclude that $E^{\prime}$ determines $L$.

Corollary 8. Let $P$ and $P^{\prime}$ be two conowheel sets. Then $\mathrm{nb}_{\mathcal{G}}(P)=\mathrm{nb}_{\mathcal{G}}\left(P^{\prime}\right)$ for any graph class $\mathcal{G}$ if and only if $F(P)=F\left(P^{\prime}\right)$. 
Proof. We already know from Theorem 3 that the frequency vector determines the number of embeddings. For the other direction, we reconstruct the number of embracing $k$-sets by appropriately choosing the graph classes $\mathcal{G}$. After that, the frequency vector is determined by Lemma 7 .

The number of embracing triangles is equal to the number of embeddings of $K_{4}^{\cdots}$ and therefore, by our assumption, the same for both $P$ and $P^{\prime}$. We now simply generalize to $k$-wheels, i.e., $\mathcal{G}$ contains a cycle with $k$ vertices, each adjacent to one additional vertex. All that is left to observe is that the number of distinct embeddings of such a $k$-wheel on a conowheel set is the same as the number of embracing $k$-sets.

Note that, for arbitrary point sets, we can compute the number of crossing-free embeddings of such $k$-wheels in polynomial time: For $k=3$, this number is equal to the number of crossing-free embeddings of $K_{4}^{\cdots}$, which can be obtained from the frequency vector associated with each point, see Section 3.2. For $k \geq 4$, we distinguish the cases where the geometric embedding of a $k$-wheel has only three vertices on the unbounded cell and where it has more. The latter case can be dealt with by computing the number of embracing $k$-sets for each point. The former can be obtained by computing the number of triangles with $j$ points in the interior and multiplying this number by $3\left(\begin{array}{c}j \\ k-2\end{array}\right)$. This is because for every vertex of such a triangle, a path of $k-2$ points inside this triangle in radial order around that vertex gives a $k$-wheel with the triangle as the unbounded cell. For all $j$, the corresponding number of triangles can be obtained in $O\left(n^{3}\right)$ time $[4,10]$.

Unfortunately, generalizing the above approach of counting embracing $k$-sets to higher dimensions fails already in 3-space. Indeed, consider the set of non-embracing tetrahedra for a set $H \subset \mathbb{R}^{3}$ in convex position. Observe now that any such tetrahedron has either three or four edges that form a "tangent" plane through $w$.

Instead, consider a partition $B \cup \dot{\cup}=H$ defined by a plane $\phi$ through $w$ that is disjoint from $H$. Then, the set of non-embracing $k$-sets consists of those completely in $B$ and $W$, respectively, and those intersected by $\phi$. For the latter, consider the intersection of $\mathrm{CH}(A)$ of such a set $A$ with $\phi$. There is again a single "tangent" point $t=p q \cap \phi$ such that $\mathrm{CH}(A) \cap \phi$ is on one side of the line $w t$ on $\phi$. Hence, the number of embracing $k$-sets in 3 -space is

$$
\mathrm{embr}_{k}=\left(\begin{array}{c}
n \\
k
\end{array}\right)-\left(\begin{array}{c}
|B| \\
k
\end{array}\right)-\left(\begin{array}{c}
|W| \\
k
\end{array}\right)-\sum_{p q \in B \times W}\left(\begin{array}{l}
\mathrm{I}(p q) \\
k-2
\end{array}\right) .
$$

We can generalize this approach in the following way.

- Lemma 9. Let $H$ be a set of $n$ points in $\mathbb{R}^{d}$, with $H \cup\{\mathbf{0}\}$ in general position, and let $\psi$ be a generic 2-flat containing the origin. Let proj: $\mathbb{R}^{d} \rightarrow \mathbb{R}^{d-2}$ be a projection that maps all of $\psi$ to $\mathbf{0} \in \mathbb{R}^{d-2}$. Then, the number of embracing $k$-sets in $H$ is

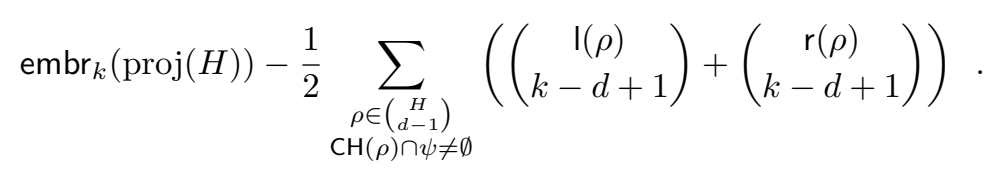

Proof. Clearly, any embracing $k$-set is also an embracing $k$-set in the projection, so we only have to subtract the number of non-embracing $k$-sets which happen to be embracing in the projection. Let $A$ be such a set. Since $\mathbf{0} \in \operatorname{proj}(\mathrm{CH}(A))$, we have $\mathrm{CH}(A) \cap \psi \neq \emptyset$. In the 2-dimensional subspace defined by $\psi$, there is a unique point $t$ on the boundary of $\mathrm{CH}(A) \cap \psi$ such that $\mathrm{CH}(A) \cap \psi$ is in the left closed halfplane defined by $0 t$ (recall that we assume general position). Since $\psi$ is generic, $t$ is the intersection of $\psi$ with a $(d-2)$-simplex defined by a tuple $\rho$ of $d-1$ points of $A$, and the oriented $(d-1)$-simplex $\mathbf{0} \rho$ has all points of $A \backslash \rho$ 
either on its positive or negative side. We are thus counting each such non-embracing $k$-set twice (for the left and the right "tangent"), and the claim follows.

With the previous lemma at hand, it is now a simple task to give a proof of our main computational result.

- Theorem 4 (restated). Let $d \geq 3$ be fixed and let $H$ be a set of $n$ points in $\mathbb{R}^{d}$. Then, the simplicial depth of a point $w$ in $H$ can be computed in $O\left(n^{d-1}\right)$ time.

Proof. The proof of Lemma 9 is constructive (apart from the choice of $\psi$, which can be done arbitrarily using the techniques in [8]). Whether a $(d-1)$-simplex intersects $\psi$ can be computed in $O\left(d^{d}\right)$ time. It thus remains to compute the values of $\mathrm{I}(\rho)$ for the $(d-1)$-tuples $\rho$. While a brute-force approach would take $O\left(n^{d}\right)$ time for this operation, we can actually consider the points of $H$ as vectors representing points in the $(d-1)$-dimensional projective plane. We compute the dual hyperplane arrangement [9] in $O\left(n^{d-1}\right)$ time, which allows us to extract the values of $\mathrm{I}(\rho)$ within the same time bounds (as also discussed in [9]).

Note that the part whose running time depends on $d$ is computing the values of $\mathrm{I}(\rho)$. After $O\left(n^{d-1}\right)$ time, we can produce a vector indicating the number of $(d-1)$-simplices intersecting $\psi$ with a certain number of points on their positive side. Using this vector, we only have to sum up over $O(n)$ binomial coefficients in each recursion step to obtain the number of embracing $k$-sets.

\subsection{Polytopes with few vertices}

Through the so-called Gale transform (cf. [31, 32, 33]), origin-embracing triangles are in correspondence to facets $((n-4)$-faces) of simplicial $(n-3)$-polytopes with at most $n$ vertices. More generally, subsets of size $i$ containing the origin in their convex hull correspond to $(n-i-1)$-faces. Therefore, some of our results connect to such simplicial $d$-dimensional polytopes with at most $d+3$ vertices (number of frequency vectors, number of order types, computation of number of embracing triangles, etc.) and thus to known results in that context.

Gale duality. For $n>d$, we call a matrix $A \in \mathbb{R}^{n \times d}$ legal if $A$ has full rank $d$ and $A^{\top} \mathbf{1}_{n}=\mathbf{0}_{d}$. Let $S_{A}=\left(p_{1}, p_{2}, \ldots, p_{n}\right)$ be the sequence of points in $\mathbb{R}^{d}$ with the coordinates of $p_{i}$ obtained from the $i$ th row of $A$. Legal thus means that $S_{A}$ is not contained in a hyperplane and that the origin is the centroid of $S_{A}$. For legal matrices $A \in \mathbb{R}^{n \times d}$ and $B \in \mathbb{R}^{n \times n-d-1}$, we call $B$ an orthogonal dual of $A$, in symbols $A \perp B$, if $A^{\top} B=\mathbf{0}_{d \times(n-d-1)} . S_{B}$ is called a Gale dual (Gale transform, Gale diagram) of $S_{A} \cdot{ }^{5}$ In other words, if $A \perp B$ then all column vectors of $B$ are orthogonal to all column vectors of $A$; together with the legal condition, this means that the column vectors of $B$ span the space of all vectors orthogonal to the column vectors of $A$ and to $\mathbf{1}_{n}$, i.e., it is a basis of the null space of the vector space spanned by the columns of $\left(A \mathbf{1}_{n}\right)$. (The matrix $\left(A \mathbf{1}_{n}\right)$ is the matrix $A$ with an extra column of 1 's.)

- Proposition 10 ([20, p. 111]). Let $A \perp B$ with $S_{A}=\left(p_{1}, p_{2}, \ldots, p_{n}\right), S_{B}=\left(p_{1}^{*}, p_{2}^{*}, \ldots, p_{n}^{*}\right)$. For every $I \subseteq[n]$, the set $\left\{p_{i} \mid i \in I\right\}$ is contained in a facet of $\mathrm{CH}\left(S_{A}\right)$ iff $\left\{p_{i}^{*} \mid i \notin I\right\}$ is embracing.

${ }^{5}$ Following [32], we add the requirement that the origin is the centroid, in contrast to, e.g., [20, Chapter 5.6]. 
For a $d$-dimensional polytope $\mathcal{P}$, the $f$-vector of $\mathcal{P}$ is defined as $f(\mathcal{P})=\left(f_{-1}, f_{0}, \ldots, f_{d-1}\right)$, where $f_{i}(\mathcal{P})$ is the number of $i$-dimensional faces ( $i$-faces) of $\mathcal{P}$ (the empty face is the unique $(-1)$-face, 0 -faces are vertices, 1 -faces are edges, $\ldots,(d-1)$-faces are facets). A property of the Gale dual is that the points in $S_{A}$ are in general position iff the rows in $B$ are linearly independent [20, p. 111]. Thus, if $S:=\left\{p_{1}, p_{2}, \ldots, p_{n}\right\}$ is a set of $n$ points in general position, $\mathcal{P}:=\mathrm{CH}(S)$, and $Q$ is the set $\left\{p_{1}^{*}, p_{2}^{*}, \ldots, p_{n}^{*}\right\}$, then $f_{i}(\mathcal{P})$ equals the number of embracing $(n-i-1)$-sets in $Q .{ }^{6}$ Computing the $f$-vector can thus be done by computing the Gale dual and by using Proposition 10.

- Proposition 11. Given a legal matrix $A \in \mathbb{R}^{n \times d}$, an orthogonal dual can be computed in time $O\left(n^{\omega}\right)$, where $\omega$ is the exponent for matrix multiplication over $\mathbb{R}$.

- Corollary 12. For a set $S$ of $n=d+k$ points in general position in $\mathbb{R}^{d}$, the number of facets of the simplicial polytope $\mathrm{CH}(S)$ can be computed in time $O\left(n^{k-2}\right)$ for $k \geq 5$ and in $O\left(n^{\omega}\right)$ otherwise, where $\omega$ is the exponent for matrix multiplication over $\mathbb{R}$.

Note that the asymptotic number of facets may be as large as $n^{k}$. A generalization of Corollary 12 to sets not necessarily in general position is possible for $k=3$. Our efficient computation of the number of embracing $k$-sets thus lets us obtain not only the $f$-vector of a polytope, but of course related vectors like the $h$ - and the $g$-vector. We finally draw the connection between order types of conowheel sets and the combinatorial structure of simplicial $d$-polytopes with $d+3$ vertices.

- Proposition 13. The family of embracing triangles of a conowheel set $P=H \cup\{w\}$ determines the order type of $P$.

It is therefore no coincidence that the number obtained in Theorem 1 is the same as the one obtained by Perles for the number of simplicial $d$-polytopes with $d+3$ vertices (see $[15$, Chapter 6.3]). Also, the number of $f$-vectors of polytopes with at most $d+3$ vertices, as obtained by Linusson [17], equals the number of frequency vectors by Lemma 7; via the Gale dual, we thus obtain a direct proof for the number of these $f$-vectors, as desired by Linusson. Doing so for $d+4$ vertices, however, remains an open problem.

\section{References}

1 Bernardo M. Ábrego and Silvia Fernández-Merchant. A lower bound for the rectilinear crossing number. Graphs Combin., 21(3):293-300, 2005. doi:10.1007/s00373-005-0612-5.

2 Peyman Afshani, Donald R. Sheehy, and Yannik Stein. Approximating the simplicial depth. CoRR, abs/1512.04856, 2015.

3 Oswin Aichholzer, Thomas Hackl, Clemens Huemer, Ferran Hurtado, Hannes Krasser, and Birgit Vogtenhuber. On the number of plane geometric graphs. Graphs Combin., 23:67-84, 2007. doi:10.1007/s00373-007-0704-5.

4 Esther M. Arkin, Samir Khuller, and Joseph S. B. Mitchell. Geometric knapsack problems. Algorithmica, 10(5):399-427, 1993. doi:10.1007/BF01769706.

5 Andries E. Brouwer. The enumeration of locally transitive tournaments. Technical Report ZW 138/80, Mathematisch Centrum, Amsterdam, 1980.

6 Andrew Y. Cheng and Ming Ouyang. On algorithms for simplicial depth. In Proc. $13^{\text {th }}$ Canadian Conference on Computational Geometry, pages 53-56, 2001.

${ }^{6}$ Note that linear independence of the rows of $B$ does not assure general position of $Q$, but projecting $Q$ to the unit circle does, as no two points are collinear with $\mathbf{0}$. 
7 Serge Dulucq and Jean-Guy Penaud. Cordes, arbres et permutations. Discr. Math., 117(1):89-105, 1993. doi : 10.1016/0012-365X (93)90326-0.

8 Herbert Edelsbrunner and Ernst P. Mücke. Simulation of simplicity: a technique to cope with degenerate cases in geometric algorithms. ACM Trans. Graph., 9(1):66-104, 1990. doi : 10.1145/77635.77639.

9 Herbert Edelsbrunner, Joseph O'Rourke, and Raimund Seidel. Constructing arrangements of lines and hyperplanes with applications. SIAM J. Comput., 15(2):341-363, 1986. doi: $10.1137 / 0215024$.

10 David Eppstein, Mark H. Overmars, Günter Rote, and Gerhard J. Woeginger. Finding minimum area k-gons. Discr. Comput. Geom., 7:45-58, 1992. doi:10.1007/BF02187823.

11 Philippe Flajolet and Marc Noy. Analytic combinatorics of non-crossing configurations. Discr. Math., 204(1-3):203-229, 1999. doi:10.1016/S0012-365X(98)00372-0.

12 Joseph Gil, William L. Steiger, and Avi Wigderson. Geometric medians. Discr. Math., 108(1-3):37-51, 1992. doi : 10.1016/0012-365X (92) 90658-3.

13 Jacob E. Goodman and Richard Pollack. Multidimensional sorting. SIAM J. Comput., 12(3):484-507, 1983.

14 Jacob E. Goodman and Richard Pollack. Semispaces of configurations, cell complexes of arrangements. J. Combin. Theory Ser. A, 37(3):257-293, 1984.

15 Branko Grünbaum. Convex Polytopes. Springer, 2nd edition, 2003.

16 Samir Khuller and Joseph S. B. Mitchell. On a triangle counting problem. Inf. Process. Lett., 33(6):319-321, 1990. doi:10.1016/0020-0190(90)90217-L.

17 Svante Linusson. The number of $M$-sequences and $f$-vectors. Combinatorica, 19(2):255-266, 1999. doi:10.1007/s004930050055.

18 R. Y. Liu. On a notion of data depth based on random simplices. Annals of Statistics, 18:405-414, 1990.

19 László Lovász, Katalin Vesztergombi, Uli Wagner, and Emo Welzl. Convex quadrilaterals and $k$-sets. In Towards a Theory of Geometric Graphs, pages 139-148. AMS, Providence, 2004.

20 Jiří Matoušek. Lectures on Discrete Geometry. Springer, 2002.

21 Juan José Montellano-Ballesteros and Ricardo Strausz. Counting polytopes via the Radon complex. J. Comb. Theory, Ser. A, 106(1):109-121, 2004. doi:10.1016/j.jcta.2004.01. 005.

22 Theodore S. Motzkin. Relations between hypersurface cross ratios, and a combinatorial formula for partitions of a polygon, for permanent preponderance, and for non-associative products. Bull. Amer. Math. Soc., 54(4):352-360, 1948.

23 Edgar M. Palmer and Robert W. Robinson. Enumeration of self-dual configurations. Pacific J. Math., 110(1):203-221, 1984.

24 Dana Randall, Günter Rote, Francisco Santos, and Jack Snoeyink. Counting triangulations and pseudo-triangulations of wheels. In Proc. $13^{\text {th }}$ Canadian Conference on Computational Geometry, pages 149-152, 2001.

25 Peter J. Rousseeuw and Ida Ruts. Bivariate location depth. J. Royal Stat. Soc. Ser. C, 45(4):516-526, 1996.

26 Andres J. Ruiz-Vargas and Emo Welzl. Crossing-free perfect matchings in wheel point sets. Unpublished manuscript, September 2015.

27 Micha Sharir and Adam Sheffer. Counting triangulations of planar point sets. Electr. J. Combin., 18(1), 2011.

28 Micha Sharir, Adam Sheffer, and Emo Welzl. Counting plane graphs: Perfect matchings, spanning cycles, and Kasteleyn's technique. J. Comb. Theory Ser. A, 120(4):777-794, 2013. doi:10.1016/j.jcta.2013.01.002. 
29 Micha Sharir and Emo Welzl. On the number of crossing-free matchings, cycles, and partitions. SIAM J. Comput., 36(3):695-720, 2006. doi:10.1137/050636036.

30 Uli Wagner. On the rectilinear crossing number of complete graphs. In Proc. $14^{\text {th }}$ Annual Symposium on Discrete Algorithms, pages 583-588. ACM/SIAM, 2003.

31 Uli Wagner and Emo Welzl. A continuous analogue of the Upper Bound Theorem. Discr. Comput. Geom., 26(2):205-219, 2001. doi:10.1007/s00454-001-0028-9.

32 Emo Welzl. Entering and leaving j-facets. Discr. Comput. Geom., 25(3):351-364, 2001. doi: $10.1007 /$ s004540010085.

33 Günter M. Ziegler. Lectures on Polytopes. Springer, 1995. 